\title{
Comparative study on Modeling of bubble bath and non bubble bath Liwen Peng
}

School of North China Electric Power University, Hebei 071003, China

2537531989@qq.com.

Keyword : Finite difference VOF Fluent

\begin{abstract}
Before comparing the heat dissipation of bubble bath and common bath, we analyze the temperature distribution in the bath.After that, we establish the heat conduction differential equation. The temperature distribution in the whole bathtub with hot water injection is obtained by the finite difference method. From the figure we can clearly see the temperature distribution in the bath is inhomogeneous. Then, we set up the model of the human body in the bathtub by using the finite element analysis of Fluent .We divide the grid, boundary conditions, and then use VOF (volume of fluid)to solve the problem. We can clearly see that the heat preservation effect with the bubble is better.
\end{abstract}

\section{1、 Introduction}

As we know, When taking a bath, we usually add the bath agent to help clean, so the analysis and study of bubble bath is very necessary. An extended model of problem based on the space factor. Because there are hot convection inside the bath when injected into hot water, which is a three-dimensional steady state, have a heat source, inconstant property, so we set up differential equations and solve this problem by the finite difference method.

\section{2、The temperature distribution in bathtub}

\section{1、 The differential equations and boundary conditions ${ }^{[1]}$}

The differential equations:

$$
0=\frac{\partial}{\partial x}\left(\lambda \frac{\partial t}{\partial x}\right)+\frac{\partial}{\partial y}\left(\lambda \frac{\partial t}{\partial y}\right)+\frac{\partial}{\partial z}\left(\lambda \frac{\partial t}{\partial z}\right)+\dot{\Phi}
$$

Boundary conditions: Between the water and the air is convection heat transfer and evaporation dissipation. Between the water and inner wall of bathtub is convection heat transfer.

$$
\begin{array}{cc}
-\lambda \frac{\partial t}{\partial x}=h_{i}\left(t_{f}-t_{w i}\right) & x=0, x=a \\
-\lambda \frac{\partial t}{\partial y}=h_{j}\left(t_{f}-t_{w j}\right) & y=0, y=b \\
-\lambda \frac{\partial t}{\partial z}=h_{k}\left(t_{f}-t_{w k}\right) & z=0
\end{array}
$$

Where $\mathrm{K}$ is the composite heat transfer coefficient .

\section{2、 The solution by finite[2] difference method}

We divide the water area of the bathtub into a number of non-overlapping sub regions, based on the conservation of energy, the node equation is obtained as follows:

$$
\begin{aligned}
& \lambda \Delta y \frac{t_{m-1 . n}-t_{m, n}}{\Delta x}+\lambda \Delta y \frac{t_{m+1 . n}-t_{m, n}}{\Delta x}+\lambda \Delta x \frac{t_{m, n+1}-t_{m, n}}{\Delta y}+\lambda \Delta x \frac{t_{m, n-1}-t_{m, n}}{\Delta y}+(\Delta x \Delta y) \dot{\Phi}=0 \\
& \lambda \frac{\Delta y}{2} \frac{t_{M-1, N}-t_{M, N}}{\Delta x}+h \frac{\Delta y}{2}\left(t_{\infty}-t_{M, N}\right)+\lambda \frac{\Delta x}{2} \frac{t_{M, N-1}-t_{M, N}}{\Delta y}+\frac{\Delta x \Delta y}{4} \dot{\Phi}=0
\end{aligned}
$$


The first one is the center node equation, the second one is the boundary node equation.

\section{3、The result}

The result of using MATLAB is as follow:

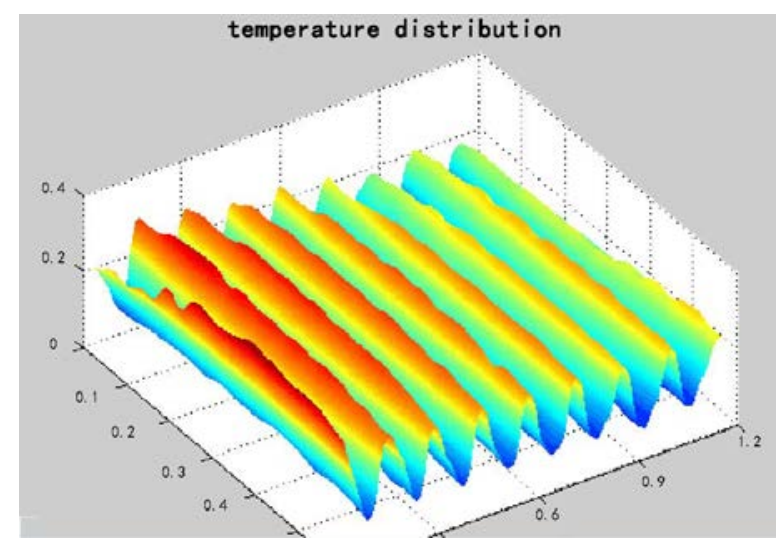

Figure 1 Temperature-Space

\section{4、 The Bubble Bath[3]}

\subsection{1、 Calculation of heat dissipation}

Soap bubbles can prevent that the water molecules penetrate the surface effectively.So the bubble model increases the thermal resistance of thermal resistance in the process of heat dissipation, and reduces the evaporation dissipation.

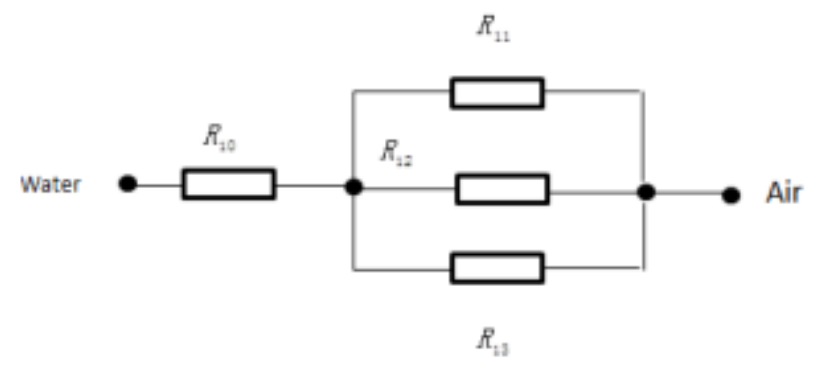

Figure 2 Hot-path-bubble

The calculation process is the same as the model 1,The film of Soap bubble is about $100 \mathrm{~nm}$, the thermal conductivity is 0.025 .According to the thermal resistance formula: $R=\frac{\delta}{K A}$.After calculation, the thicker the soap bubble is, the better the effect of heat preservation.

\subsection{2、Simulation by fluent}

We set up the model of the human body in the bathtub by using the finite element analysis of Fluent .We divide the grid, boundary conditions, and then use VOF (of Fluid Volume) to solve the problem. In the same conditions, the temperature distribution of the bath and the bubble bath are as follows: 


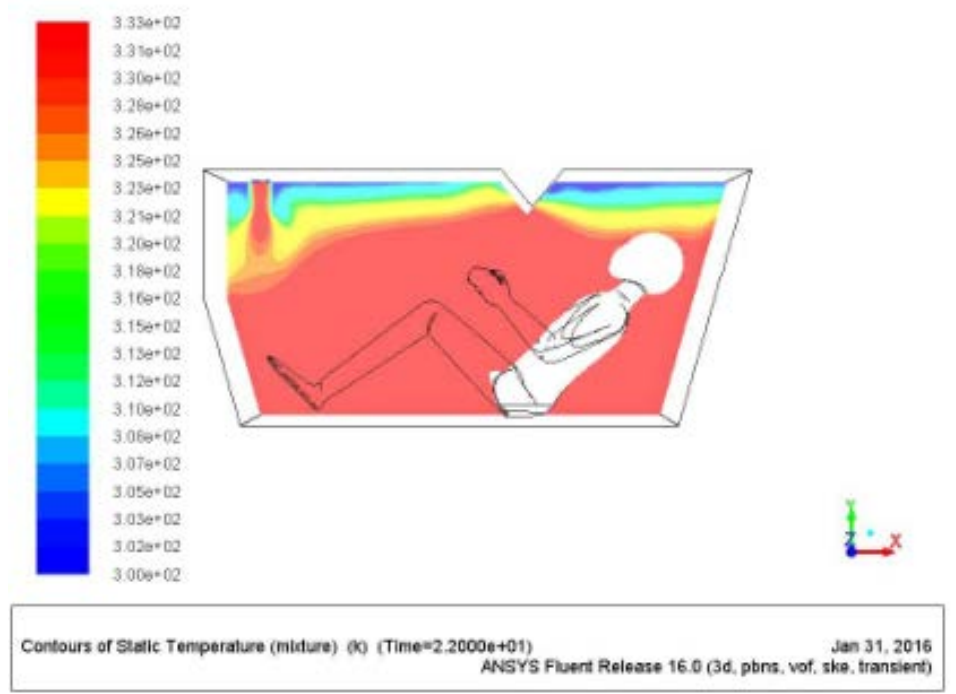

Figure 3 Temperature-no bubble

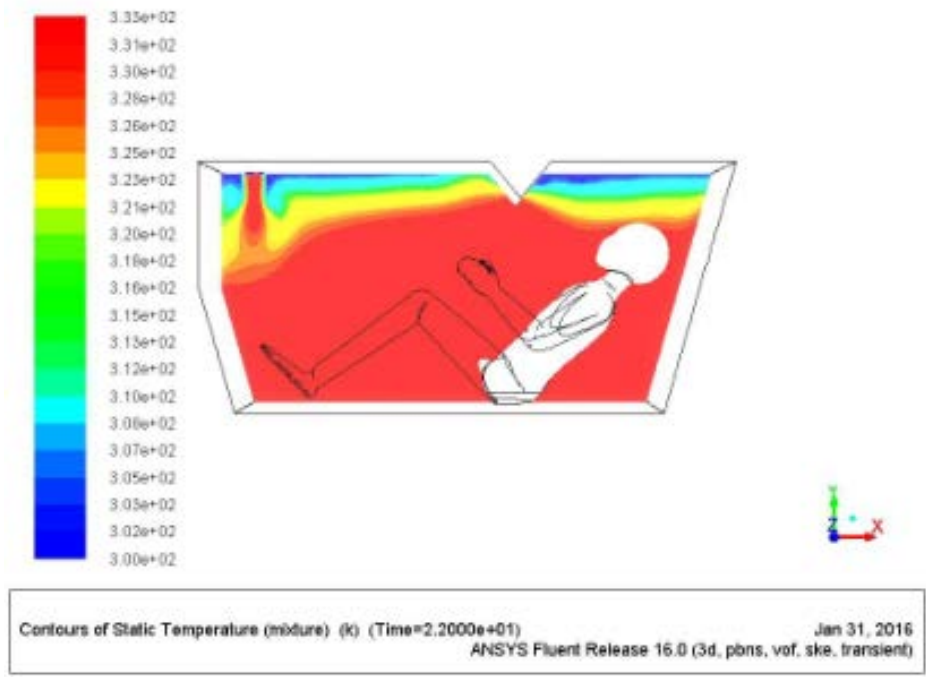

Figure 4 Temperature- bubble

\section{Summary}

The Figure 9 is not a bubble bath, the Figure 10 is a bubble bath. We can clearly see that the temperature is higher with the bubble bath, the heat preservation effect is better.

\section{References}

[1]Yonghe Zhao, Dianrong Gao,Qiguo Liang,Minggao Yin, Boundary condition treatment in finite element analysis of fluid mechanics[J], Journal of Northeast Heavy Machinery Institute,1997.

[2]Yueping Wang, numerical simulation of thermal stress in casting process base on finite difference method[D], Harbin Institute of Technology,7,2013.

[3] Effect of soap bubbles on water temperature, Feb. 4, 2015. https: //www. zhihu.com /question/ 39958914 